\title{
The polynomial part of a restricted partition function related to the Frobenius problem
}

\author{
Matthias Beck \\ Department of Mathematical Sciences \\ State University of New York \\ Binghamton, NY 13902-6000, USA \\ matthias@math.binghamton.edu
}

\author{
Ira M. Gessel * \\ Department of Mathematics \\ Brandeis University \\ Waltham, MA 02454-9110, USA \\ gessel@brandeis.edu
}

\author{
Takao Komatsu \\ Faculty of Education \\ Mie University \\ Mie, 514-8507, Japan \\ komatsu@edu.mie-u.ac.jp
}

Submitted: May 29, 2001; Accepted: September 4, 2001

MR Subject Classifications: Primary 05A15; Secondary 11P81, 05A17

\begin{abstract}
Given a set of positive integers $A=\left\{a_{1}, \ldots, a_{n}\right\}$, we study the number $p_{A}(t)$ of nonnegative integer solutions $\left(m_{1}, \ldots, m_{n}\right)$ to $\sum_{j=1}^{n} m_{j} a_{j}=t$. We derive an explicit formula for the polynomial part of $p_{A}$.
\end{abstract}

Let $A=\left\{a_{1}, \ldots, a_{n}\right\}$ be a set of positive integers with $\operatorname{gcd}\left(a_{1}, \ldots, a_{n}\right)=1$. The classical Frobenius problem asks for the largest integer $t$ (the Frobenius number) such that

$$
m_{1} a_{1}+\cdots+m_{n} a_{n}=t
$$

has no solution in nonnegative integers $m_{1}, \ldots, m_{n}$. For $n=2$, the Frobenius number is $\left(a_{1}-1\right)\left(a_{2}-1\right)-1$, as is well known, but the problem is extremely difficult for $n>2$. (For surveys of the Frobenius problem, see [R, Se].) One approach [BDR, I, K, SÖ] is to study the restricted partition function $p_{A}(t)$, the number of nonnegative integer solutions $\left(m_{1}, \ldots, m_{n}\right)$ to $\sum_{j=1}^{n} m_{j} a_{j}=t$, where $t$ is a nonnegative integer. The Frobenius number is the largest integral zero of $p_{A}(t)$. Note that, in contrast to the Frobenius problem, in the definition of $p_{A}$ we do not require $a_{1}, \ldots, a_{n}$ to be relatively prime. In the following, $a_{1}, \ldots, a_{n}$ are arbitrary positive integers.

\footnotetext{
*Research partially supported by NSF grant DMS-9972648.
} 
It is clear that $p_{A}(t)$ is the coefficient of $z^{t}$ in the generating function

$$
G(z)=\frac{1}{\left(1-z^{a_{1}}\right) \cdots\left(1-z^{a_{n}}\right)} .
$$

If we expand $G(z)$ by partial fractions, we see that $p_{A}(t)$ can be written in the form

$$
\sum_{\lambda} P_{A, \lambda}(t) \lambda^{t}
$$

where the sum is over all complex numbers $\lambda$ such that $\lambda^{a_{i}}=1$ for some $i$, and $P_{A, \lambda}(t)$ is a polynomial in $t$. The aim of this paper is to give an explicit formula for $P_{A, 1}(t)$, which we denote by $P_{A}(t)$ and call the polynomial part of $p_{A}(t)$. It is easy to see that $P_{A}(t)$ is a polynomial of degree $n-1$. (More generally, the degree of $P_{A, \lambda}(t)$ is one less than the number of values of $i$ for which $\lambda^{a_{i}}=1$.) It is well known [PS, Problem 27] that

$$
p_{A}(t)=\frac{t^{n-1}}{(n-1) ! a_{1} \cdots a_{n}}+O\left(t^{n-2}\right) .
$$

Our theorem is a refinement of this statement. We note that Israilov derived a more complicated formula for $P_{A}(t)$ in $[\mathrm{I}]$.

Let us define $Q_{A}(t)$ by $p_{A}(t)=P_{A}(t)+Q_{A}(t)$. From the partial fraction expansion above, it is clear that $Q_{A}$ (and hence also $p_{A}$ ) is a quasi-polynomial, that is, an expression of the form

$$
c_{d}(t) t^{d}+\cdots+c_{1}(t) t+c_{0}(t)
$$

where $c_{0}, \ldots, c_{d}$ are periodic functions in $t$. (See, for example, Stanley [St, Section 4.4], for more information about quasi-polynomials.) In the special case in which the $a_{i}$ are pairwise relatively prime, each $P_{A, \lambda}(t)$ for $\lambda \neq 1$ is a constant, and thus $Q_{A}(t)$ is a periodic function with average value 0 , and this property determines $Q_{A}(t)$, and thus $P_{A}(t)$. Discussions of $Q_{A}(t)$ can be found, for example, in [BDR, I, K].

We define the Bernoulli numbers $B_{j}$ by

$$
\frac{z}{e^{z}-1}=\sum_{j \geq 0} B_{j} \frac{z^{j}}{j !}
$$

(so $B_{0}=1, B_{1}=-\frac{1}{2}, B_{2}=\frac{1}{6}, B_{4}=-\frac{1}{30}$, and $B_{n}=0$ if $n$ is odd and greater than 1 .)

\section{Theorem.}

$$
\begin{aligned}
P_{A}(t)= & \frac{1}{a_{1} \cdots a_{n}} \sum_{m=0}^{n-1} \frac{(-1)^{m}}{(n-1-m) !} \sum_{k_{1}+\cdots+k_{n}=m} a_{1}^{k_{1}} \cdots a_{n}^{k_{n}} \frac{B_{k_{1}} \cdots B_{k_{n}}}{k_{1} ! \cdots k_{n} !} t^{n-1-m} \\
= & \frac{1}{a_{1} \cdots a_{n}} \sum_{m=0}^{n-1} \frac{(-1)^{m}}{(n-1-m) !} \\
& \times \sum_{k_{1}+2 k_{2} \cdots+m k_{m}=m} \frac{(-1)^{k_{2}+\cdots+k_{m}}}{k_{1} ! \cdots k_{m} !}\left(\frac{B_{1} s_{1}}{1 \cdot 1 !}\right)^{k_{1}} \cdots\left(\frac{B_{m} s_{m}}{m \cdot m !}\right)^{k_{m}} t^{n-1-m},
\end{aligned}
$$


where $s_{i}=a_{1}^{i}+\cdots+a_{n}^{i}$.

As an intermediate step, we first prove the following more elegant but less explicit formula for $P_{A}(t)$.

Proposition. $P_{A}(t)$ is the constant term in $z$ in

$$
-\frac{z e^{-t z}}{\left(1-e^{a_{1} z}\right) \cdots\left(1-e^{a_{n} z}\right)} .
$$

Proof. As noted earlier, $p_{A}(t)$ is the coefficient of $z^{t}$ in the generating function

$$
G(z)=\frac{1}{\left(1-z^{a_{1}}\right) \cdots\left(1-z^{a_{n}}\right)} .
$$

Hence if we let $f(z)=G(z) / z^{t+1}$ then $p_{A}(t)=\operatorname{Res}(f(z), z=0)$. As in [BDR], we use the residue theorem to derive a formula for $p_{A}(t)$. Since clearly $\lim _{R \rightarrow \infty} \int_{|z|=R} f(z) d z=0$,

$$
p_{A}(t)=-\operatorname{Res}(f(z), z=1)-\sum \operatorname{Res}(f(z), z=\lambda) .
$$

Here the sum is over all nontrivial $a_{1}, \ldots, a_{n}$ th roots of unity $\lambda$. It is not hard to see that $\operatorname{Res}(f(z), z=\lambda)$ may be expressed in the form $u_{\lambda}(t) \lambda^{-t}$ for some polynomial $u_{\lambda}(t)$, and thus it follows from our earlier discussion that $-\operatorname{Res}(f(z), z=\lambda)=P_{A, \lambda^{-1}}(t) \lambda^{-t}$. In particular,

$$
P_{A}(t)=-\operatorname{Res}(f(z), z=1) .
$$

To compute this residue, note that

$$
\operatorname{Res}(f(z), z=1)=\operatorname{Res}\left(e^{z} f\left(e^{z}\right), z=0\right),
$$

so that

$$
P_{A}(t)=-\operatorname{Res}\left(\frac{e^{-t z}}{\left(1-e^{a_{1} z}\right) \cdots\left(1-e^{a_{n} z}\right)}, z=0\right) .
$$

Proof of the theorem. The coefficient of $t^{n-1-m}$ in $P_{A}(t)$ is by (4) the coefficient of $z^{-n+m}$ in

$$
\frac{(-1)^{n-m}}{(n-1-m) !} \cdot \frac{1}{\left(1-e^{a_{1} z}\right) \cdots\left(1-e^{a_{n} z}\right)}
$$

which is the coefficient of $z^{m}$ in

$$
\frac{(-1)^{m}}{(n-1-m) ! a_{1} \cdots a_{n}} B\left(a_{1} z\right) \cdots B\left(a_{n} z\right),
$$

where $B(z)=z /\left(e^{z}-1\right)$, and this implies (2).

To prove (3), we first note that

$$
\log \left(\frac{z}{e^{z}-1}\right)=\sum_{j \geq 1}(-1)^{j-1} \frac{B_{j}}{j} \frac{z^{j}}{j !}
$$


as can easily be proved by differentiating both sides. Then

$$
\begin{aligned}
B\left(a_{1} z\right) \cdots B\left(a_{n} z\right) & =\exp \sum_{j \geq 1}(-1)^{j-1} \frac{B_{j} s_{j}}{j} \frac{z^{j}}{j !} \\
& =\prod_{j \geq 1} \exp \left((-1)^{j-1} \frac{B_{j} s_{j}}{j} \frac{z^{j}}{j !}\right) .
\end{aligned}
$$

Since $B_{2 i+1}=0$ for $i>0,(-1)^{j-1} B_{j}=-B_{j}$ for $j>1$, and (3) follows from (5) and (6).

Remark. It is possible to avoid the use of complex analysis and give a purely formal power series proof of the theorem. We indicate here how this can be done. We work with formal Laurent series, which are power series with finitely many negative powers of the variable. If $F(z)=\sum_{i} u_{i} z^{i}$ is a formal Laurent series $\left(u_{i}\right.$ is nonzero for only finitely many negative values of $i$ ) then the residue of $F(z)$ is res $F(z)=u_{-1}$. An elementary fact about formal Laurent series is the change of variables formula for residues: If $g(z)$ is a formal power series with $g(0)=0$ and $g^{\prime}(0) \neq 0$ then

$$
\operatorname{res} F(z)=\operatorname{res} F(g(z)) g^{\prime}(z) \text {. }
$$

(See, for example, Goulden and Jackson [GJ, p. 15].)

By partial fractions, we have

$$
G(z)=\frac{1}{\left(1-z_{1}^{a}\right) \cdots\left(1-z^{a_{m}}\right)}=\frac{c_{1}}{1-z}+\cdots+\frac{c_{m}}{(1-z)^{m}}+R(z),
$$

where $R(z)$ is a rational function of $z$ with denominator not divisible by $1-z$. It follows from our earlier discussion that

$$
\sum_{t=0}^{\infty} P_{A}(t) z^{t}=\frac{c_{1}}{1-z}+\cdots+\frac{c_{m}}{(1-z)^{m}}
$$

and thus

$$
P_{A}(t)=\sum_{l=1}^{\infty} c_{l}\left(\begin{array}{c}
t+l-1 \\
l-1
\end{array}\right)
$$

where we take $c_{l}$ to be 0 for $l>m$. Now let $U(z)=G(1-z)$. Then

$$
\begin{aligned}
U(z) & =\frac{1}{\left(1-(1-z)^{a_{1}}\right) \cdots\left(1-(1-z)^{a_{m}}\right)} \\
& =\frac{c_{1}}{z}+\cdots+\frac{c_{m}}{z^{m}}+R(1-z),
\end{aligned}
$$

where $R(1-z)$ has a formal power series expansion (with no negative powers of $z$ ), and thus $c_{l}=\operatorname{res} z^{l-1} U(z)$. Note that this holds for all $l \geq 1$, since for $l>m, c_{l}=\operatorname{res} z^{l-1} U(z)=0$. 
Then

$$
P_{A}(t)=\sum_{l=1}^{\infty} c_{l}\left(\begin{array}{c}
t+l-1 \\
l-1
\end{array}\right)=\operatorname{res} \frac{U(z)}{z} \sum_{l=1}^{m} z^{l}\left(\begin{array}{c}
t+l-1 \\
l-1
\end{array}\right)=\operatorname{res} \frac{U(z)}{(1-z)^{t+1}}
$$

We now apply the change of variables formula with $g(z)=1-e^{z}$ and we obtain

$$
\begin{aligned}
P_{A}(t) & =-\operatorname{res} \frac{U\left(1-e^{z}\right)}{e^{t z}} \\
& =-\operatorname{res} \frac{e^{-t z}}{\left(1-e^{a_{1} z}\right) \cdots\left(1-e^{a_{m} z}\right)},
\end{aligned}
$$

which is (4), and the proof continues as before.

\section{References}

[BDR] M. Beck, R. DiAz, S. Robins, The Frobenius problem, rational polytopes, and Fourier-Dedekind sums, to appear in J. Number Theory.

[GJ] I. P. Goulden And D. M. Jackson, Combinatorial Enumeration, Wiley, New York, 1983.

[I] M. I. IsRailov, Numbers of solutions of linear Diophantine equations and their applications in the theory of invariant cubature formulas, Sibirsk. Mat. Zh. 22 (1981), no. 2, 121-136, 237. English translation: Siberian Math. J. 22 (1981), no. 2, 260-273.

[K] T. Komatsu, The number of solutions of the Diophantine equation of Frobenius, to appear in J. Théor. Nombres Bordeaux.

[PS] G. Pólya And G. Szegő, Aufgaben und Lehrsätze aus der Analysis, Springer-Verlag, Berlin, 1925.

[R] J. L. Ramirez Alfonsin, The diophantine Frobenius problem, Report No. 00893, Forschungsinstitut für diskrete Mathematik, Universität Bonn (2000).

[Se] E. S. Selmer, On the linear diophantine problem of Frobenius, J. reine angew. Math. 293/294 (1977), 1-17.

[SÖ] S. Sertöz, A. ÖzLük, On the number of representations of an integer by a linear form, Istanbul Üniv. Fen Fak. Mat. Derg. 50 (1991), 67-77.

[St] R. P. Stanley, Enumerative Combinatorics, Vol. 1, Wadsworth \& Brooks/Cole, Monterey, California, 1986. 